\title{
Rapid screening for significant bacteriuria using a Coulter Counter
}

\author{
R. SMITHER 1
}

From the Bacteriology Department, Wright-Fleming Institute, St. Mary's Hospital Medical School, London W2 IPG, UK

SUMMARY A Coulter Counter was evaluated for detecting significant bacteriuria. Simple urinary particle counts showed agreement in 883 of the $956(92.4 \%)$ urine specimens examined compared to a standard culture procedure. They also provided a semiquantitative estimate of bacterial numbers in specimens diagnosed as infected by culture. Sample preparation before counting was minimal, and results were obtained within two to four minutes.

The determination of significant bacteriuria $\left(\geqslant 10^{5}\right.$ bacteria/ml of urine) by the colony count procedure (Kass, 1956) is laborious, and in consequence many other techniques have been investigated. Because of inconsistent results, methods involving 2,3,5-triphenyltetrazolium chloride (Simmons and Williams, 1962), nitrate reductase (Kahler and Guze, 1957), catalase (Braude and Berkowitz, 1961), and glucose oxidase (Scherstén et al., 1968) appear to have been abandoned in favour of semiquantitative bacteriological tests in which calibrated loops (Hoeprich, 1960) and dip-slides (Mackey and Sandys, 1965) are used. Recently, the estimation of bacterial adenosine 5'-triphosphate (ATP) in urine by instrumental analysis (Alexander et al., 1976; Johnston et al., 1976) has added a new approach to the detection of significant bacteriuria, due to the speed with which results can be obtained.

This paper describes a study in which the Coulter Counter was tested to determine its suitability for the rapid screening of urines for significant bacteriuria.

\section{Material and methods}

\section{CULTURE METHOD}

A total of 956 urine specimens, mainly from male and female inpatients, were studied. The specimens were those received in a diagnostic bacteriological service and were stored at $4^{\circ} \mathrm{C}$ before examination by

${ }^{1}$ Present address: Laboratory of the Government Chemist, Department of Industry, Cornwall House, Stamford Street, London SE1 9NQ, UK

Received for publication 2 June 1977 the filter paper count method of Ryan et al. (1962). In this test, the end $\frac{1}{2}$ inch of a 3 inch long $\times \frac{1}{4}$ inch wide sterile filter paper strip is immersed in the urine. After excess urine has been removed, the strip is placed in contact with the surface of an agar pou plate, where the number of colonies formed related directly to the number of viable organisms present in the urine. Six duplicated urine samples were accommodated on a MacConkey agar plate, the colonies being counted after 18-24 hours' incubation at $37^{\circ} \mathrm{C}$. Significant bacteriuria was considered present when an average of 25 or more colonies, in pure culture, developed in each area of filter paper/agar contact. Culture negative specimens included those with 'no significant growth'.

\section{PREPARATION OF URINES FOR COULTER} COUNTS

Preliminary investigations had shown that when specimens were allowed to stand for 30 minutes, the bacterial population of the urine near the surface remained relatively constant while the numbers of other particles decreased. This elimination of large particles minimised the likelihood of an orifice blockage and subsequently reduced the incidence of false results. Following culture and the period of settlement, a $0.1 \mathrm{ml}$ urine sample was removed from the meniscus of each specimen with an automatic pipette (Eppendorf Marburg, Hamburg, West Germany) and transferred to a clean glass specimen tube containing $19.9 \mathrm{ml}$ of particle-free $0.9 \% \mathrm{NaCl}$ (Polyfusor, Boots, Nottingham, UK) with $0.3 \%$ formaldehyde as fixative. No further dilution of specimens was usually necessary. When apparent, erythrocytes were lysed by adding three drops of 
Table 1 Analysis of 956 urine results according to filter paper count and Coulter Counter methods

\begin{tabular}{|c|c|c|c|c|c|c|}
\hline \multicolumn{2}{|c|}{ Filter paper count method } & \multicolumn{4}{|c|}{ Coulter Counter method } & \multirow{3}{*}{$\begin{array}{l}\text { Approximate viable count per } \mathrm{ml} \\
\text { of urine for culture positive } \\
\text { specimens* }\end{array}$} \\
\hline \multirow[t]{2}{*}{ Result } & \multirow[t]{2}{*}{ No. of specimens } & \multirow[t]{2}{*}{ Result } & \multicolumn{3}{|c|}{ No. of specimens } & \\
\hline & & & Totals & $T 25$ count $>6250$ & $T 25$ count $<6250$ & \\
\hline+ & $191_{+}^{+}$ & $\left\{\begin{array}{l}+ \\
-\end{array}\right.$ & $\begin{array}{r}155 \\
36\end{array}$ & $\left\{\begin{array}{l}44(\mathrm{~A}) \dagger \\
52(\mathrm{~B}) \\
37(\mathrm{C})\end{array}\right.$ & $\begin{array}{l}22 \\
36\end{array}$ & $\begin{array}{r}>10^{8} \\
10^{7}-10^{8} \\
10^{6}-10^{7} \\
10^{6}-10^{7} \\
10^{5}-10^{6}\end{array}$ \\
\hline- & 765 & $\{+$ & 37 & $\left\{\begin{array}{l}14(B) \\
23(C)\end{array}\right.$ & 683 & \\
\hline Totals & 956 & & 956 & 215 & 741 & \\
\hline
\end{tabular}

* Figures based on unpublished data from preliminary investigations

$\dagger$ Zones in which specimens occurred, based on T25 v T100 counts

‡Four specimens detected, only when repeated on blood agar

Zaponin (Coulter Electronics, Harpenden, Herts, UK) to each $20 \mathrm{ml}$ of the diluted urine. The tube was capped and inverted once to suspend the urine particles. Between samples the orifice tube was rinsed with fresh saline to avoid contamination.

\section{COUNTING AND ASSESSMENT OF URINES}

\section{FROM COUNT DATA}

Details of the instrument, a model Fn Coulter Counter, have already been described (Smither, 1975). Aperture current and attenuation settings of 8 and $0 \cdot 250$ were used, but when particularly large or small organisms were present interpretation of their pulses on the oscilloscope screen was improved by adjusting the attenuation.

Individual counts required $0.05 \mathrm{ml}$ of diluted urine and took 13 seconds to complete. An average count, recorded at a threshold setting of 25 (T25), was derived from three consecutive counts. Urines with particle counts $<6250$ were presumed negative and those with counts $>6250$ were presumed positive. In addition, a count was recorded at T100 when the T25 count was $>6250$. By manipulating the T25 and T100 counts, a substantial proportion of potentially false positive specimens (culture negative urines with T25 counts $>6250$ ) were resolved. This was aided by the experience gained in recognising distinctive oscilloscope particle pulse patterns, a process which also enabled a number of culture positive specimens with T25 counts $<6250$ to be distinguished.

Results for presumed negative urines were obtained in less than two minutes and those for presumed positive urines, which required the additional T100 counts, were obtained in three to four minutes.

\section{Results}

Of the 956 urine specimens examined for significant bacteriuria, 191 were culture positive and 765 were culture negative by the filter paper count method (Table 1). The Coulter Counter method correctly monitored 155 culture positive and 728 culture negative specimens, giving a total agreement of $92.4 \%$ compared to the culture method. However, there were $36(18.8 \%)$ false negative and $37(4.8 \%)$ false positive specimens, representing a sensitivity of $81.2 \%$ and a specificity of $95.2 \%$ relative to the culture method.

A total of 215 urines, composed of 133 culture positive and 82 culture negative specimens, had T25 counts $>6250$ and were initially presumed positive. Previous (unpublished) studies had revealed that, on average, the T25 count of a culture positive urine was considerably greater than that of a potentially false positive specimen, whereas the T100 count was much smaller. After compounding these differences by plotting T25 counts against T100 counts, the resulting points were distributed in three zones (Fig. 1). For culture positive urines the zones related directly to the number of organisms present (Table 1). All 44 specimens in zone $A$, and the majority of specimens in zone B (52 of 66), were culture positive. Only 37 of the 105 specimens in zone $\mathrm{C}$ were culture positive, however.

The 37 false positive specimens, which occurred in zones $B$ and $C$, contained regular unidentified nonbacterial particles whose displayed pulses were indistinguishable from those of bacteria. No attempt was made to identify these particles. Forty-five specimens (zone $C$ ) with T25 counts $>6250$ were recognised as being culture negative because they 


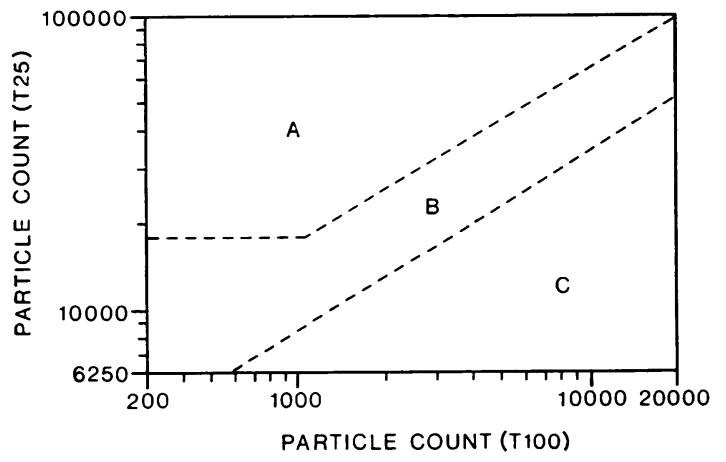

Fig. 1 Zones into which presumed positive specimens fall when T25 counts are plotted against T100 counts.

had irregular particle pulse patterns. In about half of these cases, high particle counts appeared to be correlated with non-bacterial genitourinary disorders and the use of methods by which such disorders are examined. For example, patients with ureter/renal impairment or bladder carcinomas, and men who had had prostatectomies, were predisposed to producing urines with raised particle counts. Others who were undergoing or had undergone cystoscopy, or who had been catheterised, also produced high particle counts. There was no correlation, however, between high counts and either the patient's age or sex, or the presence of proteinuria. Pregnant women were as likely to produce urines of this type as were other women.
There were 741 specimens with T25 counts $<6250$, of which 683 were culture negative (Table 1). Also in this category were 22 culture positive specimens containing of the order of $10^{6}-10^{7}$ organisms $/ \mathrm{ml}$ of urine which were identified as being positive simply from their oscilloscope patterns, whereas the 36 with only $10^{5}-10^{6}$ organisms/ $\mathrm{ml}$ were indistinguishable from culture negative specimens and were thus falsely negative.

Oscilloscope patterns of particles in three different urines are shown (Fig. 2), where the threshold was reduced to zero to display the pulses fully. The culture positive specimen (Fig. 2A), containing Escherichia coli, exhibited numerous regular bacterial cell pulses, whereas the potentially false positive specimen (Fig. 2B) displayed pulses of a predominantly heterogeneous nature with more pulses from large particles. The typical culture negative specimen (Fig. 2C) showed pulses mainly from small particles.

The organisms isolated from culture positive specimens are listed in Table 2 . Three patients with recurrent low-level urinary infections, who had not responded to various antibiotic regimes, accounted for 11 of the 36 false negative urines. Two, whose urines contained Klebsiella spp, had each given foug specimens during the test programme while the other, containing $E$. coli, had given three. Four fals se negative specimens contained Candida albicans.The Coulter method detected four infections (two with Streptococcus viridans and two with Streptococcus pyogenes) which were not detected by the filter paper count method using MacConkey agar

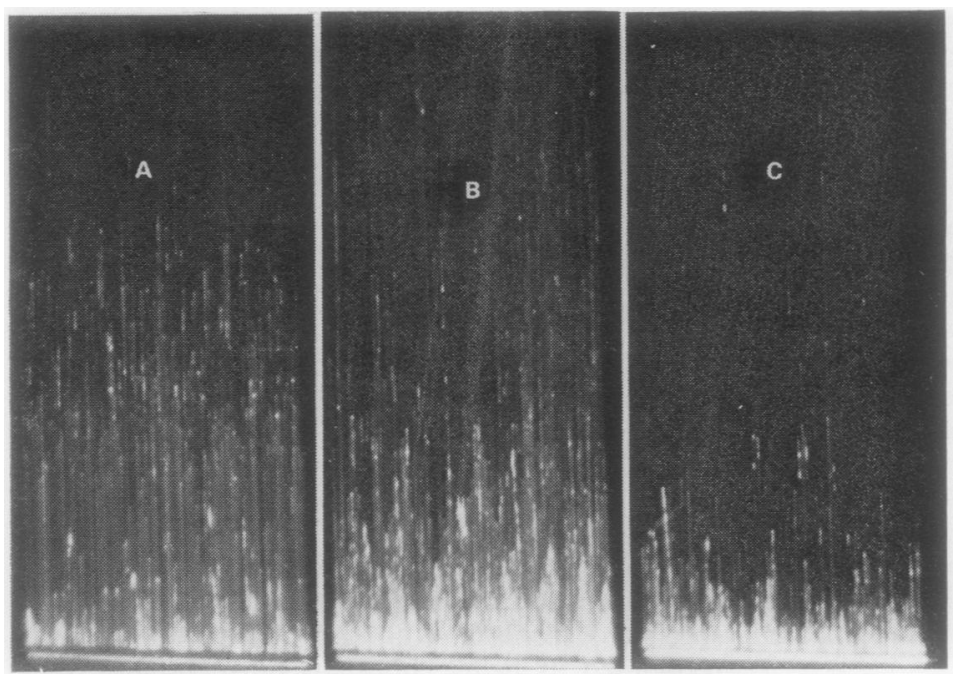

Fig. 2 Coulter Counter oscilloscope screen showing particle pulses from urine infected with $\mathrm{E}$. coli $(A)$, and potentially false positive $(B)$, and culture negative $(C)$ urines. 
Table 2 Organisms isolated from positive urine specimens as detected by the filter paper count method

\begin{tabular}{|c|c|c|c|c|}
\hline \multirow[t]{3}{*}{ Organisms } & \multicolumn{4}{|c|}{ Culture positive specimens } \\
\hline & \multicolumn{2}{|c|}{$\begin{array}{l}\text { Detected by Coulter } \\
\text { Counter }\end{array}$} & \multicolumn{2}{|c|}{$\begin{array}{l}\text { Not detected by } \\
\text { Covlter Counter }\end{array}$} \\
\hline & No. & $\%$ & No. & $\%$ \\
\hline $\begin{array}{l}\text { Escherichia coli (and other } \\
\text { coliforms) }\end{array}$ & 81 & $52 \cdot 3$ & 8 & $22 \cdot 2$ \\
\hline Pseudomonas aeruginosa & 13 & $8 \cdot 4$ & 2 & $5 \cdot 6$ \\
\hline Proteus spp & 10 & $6 \cdot 5$ & 3 & $8 \cdot 3$ \\
\hline Klebsiella spp & 7 & $4 \cdot 5$ & 9 & $25 \cdot 0$ \\
\hline Streptococcus faecalis & 6 & 3.9 & 3 & $8 \cdot 3$ \\
\hline Staphylococcus albus & 3 & 1.9 & 2 & $5 \cdot 6$ \\
\hline Staphylococcus axreus & 1 & 0.6 & 0 & $0 \cdot 0$ \\
\hline Hafnia spp & 1 & 0.6 & 0 & $0 \cdot 0$ \\
\hline Candida albicans & 0 & $0 \cdot 0$ & 4 & $11 \cdot 2$ \\
\hline Mixed cultures & 29 & $18 \cdot 7$ & 5 & $13 \cdot 9$ \\
\hline Streptococcus viridans* & 2 & $1 \cdot 2$ & 0 & $0 \cdot 0$ \\
\hline Streptococcus pyogenes* & 2 & $1 \cdot 2$ & 0 & 0.0 \\
\hline Totals & 155 & & 36 & \\
\hline
\end{tabular}

*Samples detected by Coulter Counter but not by the filter paper count method using MacConkey agar

despite viable counts of the order of $10^{8}$ organisms $/ \mathrm{ml}$ of urine. Blood agar was used to confirm bacteriuria in these specimens, where suspicion about their nature had been initially aroused during the routine microscopy of their deposits.

\section{Discussion}

With the test conditions described the method was unable to detect some specimens containing the critical range of $10^{5}-10^{6}$ organisms $/ \mathrm{ml}$ of urine because of confusion with the relative excess of other urine particles. False positive specimens also arose as a result of the method being unable to discriminate successfully between the regular pulses from certain unidentified particles and those from bacteria. It was realised that false positives could also arise from patients undergoing antibiotic treatment for significant bacteriuria, where the pulse response of non-viable organisms would be the same as that from viable cells.

During preliminary studies, various methods were examined to reduce the incidence of false results. An attempt to recover and analyse the particle fraction containing the bacteria, by passage through a series of membrane filters of decreasing pore size, was unsuccessful, as were attempts to diagnose a greater number of culture positive specimens by selectively detecting bacterial volume peaks within total urine particle volume distribution curves. Estimations of mean particle volume coupled with particle pulse height analysis, by ancillary instrumentation, were also unsuccessful.

Despite these difficulties the results compared favourably with those obtained by others using a Coulter Counter (Truant et al., 1962; Truant, 1964) and where the ATP content of urinary organisms was estimated by instrumentation (Alexander et al., 1976; Johnston et al., 1976). The most useful qualities of the Coulter technique appeared to be general method simplicity, low cost per specimen in terms of materials and preparation time, and the rapid production of results. In retrospect, it should be mentioned that with experience most urines could be categorised simply on the basis of pulse pattern recognition. The formal recording of counts in fact considerably slowed the process of obtaining results, which could otherwise have been achieved in about 30-45 seconds per specimen without substantial loss of accuracy. It was also probable that anaerobic urinary tract infections could be detected by this method.

The qualities described were likely to be outweighed by other factors, however, such as the initial cost of instrumentation, the requirement for a technician to be constantly monitoring specimens, and, most importantly, the relatively high incidence $(18.8 \%)$ of undiagnosed urinary infections. Furthermore, since the particular workload of the hospital environment is often not conducive to preparing individuals for passing clean mid-stream urines, the inevitable, often excessive, contamination that many urines received undoubtedly handicapped the instrument by increasing susceptibility to false results. Because of these problems, and especially with regard to the frequency of false negative results. it seemed unlikely that the instrument could satisfactorily screen urines originating from hospital wards. Nevertheless, where more attention is paid to cleansing genital surfaces and to examining fresh, genuine mid-stream urines, for example in screening programmes involving children and pregnant women, the rapid response of the Coulter Counter may be usefully employed. In this context the method might be streamlined by performing a total particle count for each specimen at T25 only. Although this would increase the amount of false positive results to an arguably intolerable level (in the present survey to a figure of $10.7 \%$ ), it would possibly lend the method to a degree of automation, which would obviate the need for a full-time technician. It would then allow nurses in antenatal clinics, for example, to test urines by this method while performing other urine tests simultaneously. Coulter positive specimens, as well as Coulter negative specimens from individuals showing symptoms associated with significant bacteriuria, could then be sent to the laboratory for further examination.

The advice of Dr R. E. Trotman and Professor Sir 
Robert Williams is gratefully acknowledged, as is the sponsorship from the National Fund for Research into Crippling Diseases. Thanks are extended to $\mathrm{Mr}$ Anthony Turnbull and Miss Rosalind Scutt for their co-operation in collating filter paper counts with the Coulter Counter data, and also to Mrs Doreen Irving for statistical advice.

\section{References}

Alexander, D. N., Ederer, G. M., and Matsen, J. M. (1976). Evaluation of an adenosine 5'-triphosphate assay as a screening method to detect significant bacteriuria. Journal of Clinical Microbiology, 3, 42-46.

Braude, A. I., and Berkowitz, H. (1961). Detection of urinary catalase by disk flotation. Journal of Laboratory and Clinical Medicine, 57, 490-494.

Hoeprich, P. D. (1960). Culture of the urine. Journal of Laboratory and Clinical Medicine, 56, 899-907.

Johnston, H. H., Mitchell, C. J., and Curtis, G. D. W. (1976). An automated test for the detection of significant bacteriuria. Lancet, 2, 400-402.

Kahler, R. L., and Guze, L. B. (1957). Evaluation of the Griess nitrite test as a method for the recognition of urinary tract infection. Journal of Laboratory and Clinical Medicine, 49, 934-937.
Kass, E. H. (1956). Asymptomatic infections of the urinary tract. Transactions of the Association of American Physicians, 69, 56-64.

Mackey, J. P., and Sandys, G. H. (1965). Laboratory diagnosis of infections of the urinary tract in general practice by means of a dip-inoculum transport medium. British Medical Journal, 2, 1286-1288.

Ryan, W. L., Hoody, S., and Luby, R. (1962). A simple quantitative test for bacteriuria. Journal of Urology, 88, 838-840.

Scherstén, B., Dahlqvist, A., Fritz, H., Köhler, L., and Westlund, L. (1968). Screening for bacteriuria with a test paper for glucose. Journal of the American Medical Association, 204, 113-116.

Simmons, N. A., and Williams, J. D. (1962). A simple test for significant bacteriuria. Lancet, 1, 1377-1378.

Smither, R. (1975). Use of a Coulter Counter to detect $\vec{N}$ discrete changes in cell numbers and volume during growth of Escherichia coli. Journal of Applied Bacteri- U ology, 39, 157-165.

Truant, J. P. (1964). Evaluation of various methods used for the detection of significant bacteriuria in humans. Henry Ford Hospital Medical Bulletin, 12, 237-250.

Truant, J. P., Brett, W. A., and Merckel, K. E. (1962). Application of an electronic counter in the evaluation of significant bacteriuria. Henry Ford Hospital Medical $\mathbb{\Phi}$ Bulletin, 10, 359-373. 